\title{
Assessment of studies based on the use of questionnaires in the time of Covid-19 pandemic
}

\section{Corinna Porteri ${ }^{1}$ (D)}

Accepted: 5 September 2021 / Published online: 12 September 2021

(c) Monash University 2021

\begin{abstract}
During the covid-19 pandemic a huge number of research projects have been promoted internationally, nationally and locally. This also happened in Italy which was the first affected European country. Among these studies, research protocols based on the use of online questionnaires to evaluate the reaction of health workers and researchers to the emergency situation and their state of well-being were promoted. This contribution focuses on research protocols based on the use of online questionnaires and presents as a case study three different research proposals with the purpose of showing their different degree of compliance to scientific and ethical requirements and the need for a clear regulation. A guideline for the assessment of studies based on questionnaires is then suggested, in the absence of a national regulation on the issue. Having established rules, albeit flexible, can help to guarantee transparency in the evaluation process and contribute to the scientific and ethical compliance of the research.
\end{abstract}

Keywords Covid-19 $\cdot$ Pandemic $\cdot$ Research ethics $\cdot$ Ethics committee $\cdot$ Bioethics . Guideline

\section{Introduction}

During the covid-19 pandemic a huge number of research projects have been promoted internationally, nationally and locally. This also happened in Italy which was the first affected European country.

Study proposals covered many different aspects of the infection - from research on biological materials and biobanks, to observational studies, to clinical trials for possible treatments. Research protocols based on the use of online questionnaires to

Corinna Porteri

cporteri@ fatebenefratelli.eu

1 Bioethics Unit, IRCCS Istituto Centro San Giovanni Di Dio Fatebenefratelli, Via Pilastroni, 4, 25125 Brescia, Italy 
evaluate the reaction of health workers and researchers to the emergency situation and their state of well-being were also promoted.

Research contributes to increasing knowledge in new and unknown areas and to improving the possibilities of treatment and health for individuals. Research must therefore be promoted to the highest possible degree.

At the same time, any kind of studies should be carried out in compliance with the scientific and ethical requirements on which a broad convergence has been achieved (Emmanuel et al. 2000). In fact, research subjects must be protected in their rights and society needs to be reassured on the quality of the research and the achieved results.

This also applies to emergency situations, albeit a revision of standard procedures for research protocols evaluation and an amount of flexibility are certainly justified by the need to act in exceptional time constraints and situations (WHO 2010; WHO 2020; EMA 2020; AIFA 2020).

Nowadays, there is no clear national regulation for every type of research; this is also the case for non-drug observational study in Italy (Gruppo di Lavoro ISS Bioetica COVID-19 2000).

This contribution focuses on research protocols based on the use of online questionnaires and presents as a case study three different research proposals with the purpose of showing their different degree of compliance to scientific and ethical requirements and the need for a clear regulation. A guideline for the assessment of studies based on questionnaires is then suggested, in the absence of a national regulation on the issue.

\section{Studies based on questionnaires}

Especially during the first wave of the pandemic, research protocols based on the use of online questionnaires to evaluate the reaction of health workers and researchers to the emergency situation were promoted in Italy.

This paper analyses three proposals in which an Italian institute for research and care has been invited to participate. They are representative of three different ways of managing research protocols with different degree of compliance to scientific and ethical requirements.

The three proposals were addressed to health professionals and researchers with the declared aim to collect data on resilience, stress and wellbeing during the pick of the pandemic.

A first proposal was promoted by an Italian research institute and accompanied by a two lines email just saying "we have prepared an anonymous survey on the impact of the pandemic and the measures of restrain on the resilience of researchers in Italy" and inviting to disseminate the questionnaire. The link to the online questionnaire did not contain any information on the study or consent to participation, but only the title of the research and the information that by answering the questionnaire the subject authorized the processing of data for scientific purposes. The questionnaire, formally aimed at assessing the resilience of researchers during the pandemic, contained, in addition to standard tests for assessing resilience, anxiety 
and depression, a number of questions hardly related or not related to the declared study purpose.

A second proposal was promoted by the leader of a network of Italian research institutes and addressed to those institutes. The questionnaire was accompanied by a letter in which the study rationale and aim were well described and how the questionnaire was developed starting from the DSM-5 Post Traumatic Stress Disorder checklist and adapted to the specific event of the covid-19 pandemic was explained. The link to the online questionnaire opened with an information to the potential research participants containing the description of the study (reason, aim, use of data, privacy compliance) and the possibility to accept or not accept the participation by clicking a button and filling in the questionnaire.

The third proposal was an international study promoted by a Latin American University with a national coordinator centre in every country participating in the study. The invitation to answer the questionnaire was accompanied by a full research protocol translated into Italian, with the description of the background and rationale, aims, typology of the study, participating centres, sample selection, inclusion and exclusion criteria, instruments description (ad hoc questionnaires and standard tests), statistical analysis, risk and benefit ratio, ethical aspects including informed consent, privacy, and the approval of the international promoter and national coordinator ethics committees. The informed consent was available online before starting the questionnaire and a pdf copy of the consent was downloadable.

Even though the complexity of the third study in terms of countries involved and design, which also included follow-up interviews, was higher than the first two, the three proposals represent three different ways of managing studies based on the use of online questionnaires and the related invitation to participate. In the first case no research protocol is presented and no information is provided to the subjects; in addition, data are collected that do not correspond to what the title of the project and the accompanying email declare. In the second case, no research protocol is presented, although an accompanying letter describing the study and clear information to the subjects are provided. In the last case, a detailed research protocol is presented together with clear information to the subjects and the favourable opinion of the relevant ethics committees.

\section{Proposal for a guideline}

In the absence of a national regulation regarding the scientific and ethical evaluation of studies based on the use of questionnaires, a local regulation may help to ensure that research is of social value, scientifically sound and ethically good. In this context, local ethics committees may play an important role.

The following procedure has been developed by a working group and then approved by the ethics committee (EC) of the Italian institute receiving the three above study proposals in order to promote good practices of research.

The procedure establishes which study materials should be available, and the evaluation process to be followed. 
For the dissemination of questionnaires promoted by external bodies and addressed collectively to the institute staff, the following documentation is required: (a) research protocol, clearly showing: rationale, objectives, participants, methodology, use of data, return of results; (b) questionnaire for data collection; (c) information sheet in which the study is sufficiently delineated, and consent form to participate in the study; (d) information and consent to the processing of personal data; (e) opinion of the coordinator ethics committee. Only exceptionally studies that do not present all the required documentation will be taken into consideration.

In terms of evaluation process, the study materials should be transmitted by any office that receive it to the scientific and health directors of the institute for a first evaluation that may involve, where relevant, the general director.

After the directors' clearance, the research documentation is submitted to the ethics committee, which evaluates the study following two methodologies in relation to the urgency: (1) non-urgent: the documentation is evaluated by the EC during the first scheduled meeting according to the ordinary procedure that apply to any other study; (2) urgent: the research documentation is sent electronically to a subgroup of EC members who express the acceptance or rejection of the study. The subgroup is chosen on the basis of the study specificity and includes at least: the president / vice president, the bioethics expert, the legal and privacy expert, the statistician / methodology expert. The subgroup opinion and the evaluation process are recorded in the first EC scheduled meeting. The EC opinion is forwarded to the institute directors and the study promoter.

In the case of favourable opinion, the scientific and health directors will provide to the dissemination of the questionnaire among the institute staff. The individual health worker and researcher will then decide for her/himself whether or not to participate in the study.

\section{Conclusion}

The suggested procedure is a proposal that may not fit all local situations; nevertheless, it may represent a starting point for further suggestions.

We believe that a clear and written procedure may guarantee transparency in the evaluation process and contribute to the scientific and ethical compliance of the research.

Having established rules, albeit flexible, can help to proceed effectively even in emergency and complex situations, avoiding on the one hand the risk of stopping or delaying interesting studies and on the other hand the risk of participating in studies without a clear aim, of little or no social/scientific value, and not respectful of the participants' rights.

\section{Funding None.}

Data availability N.A. 


\section{Declarations}

Conflict of interest Author declare that no conflict of interest.

\section{References}

AIFA - Agenzia Italiana del Farmaco (2020) Valutazione degli studi sull'infezione da SARS-CoV-2 da parte dei Comitati Etici. Roma, 25 maggio 2020. Available at: https://www.aifa.gov.it/-/valutazione-degli-studi-sull-infezione-da-sars-cov-2-da-parte-dei-comitati-etici [Last accessed 14 October 2020].

Emanuel, E.J., D. Wendler, and C. Grady (2000) What makes clinical research ethical? JAMA 283: 2701-2711.

EMA -European Medical Agency (2020) Guidance on the management of clinical trials during the covid19 (coronavirus) pandemic. Version 3; 28 April 2020. Available at: https:/ec.europa.eu/health/sites/ health/files/files/eudralex/vol-10/guidanceclinicaltrials_covid19_en.pdf [Last accessed 14 October 2020].

Gruppo di Lavoro ISS Bioetica COVID-19 (2020) Etica della ricerca durante la pandemia di COVID-19: studi osservazionali e in particolare epidemiologici. Versione del 29 maggio 2020. Roma: Istituto Superiore di Sanità (Rapporto ISS COVID-19 n. 47/2020). Available at: https://www.iss.it/docum ents/20126/0/Rapporto+ISS+COVID-19+47_2020+\%281\%29.pdf/6cd3f35b-7c44-2cb1-6b05b3f5ac5c6870?t=1594707862248 [Last accessed 14 October 2020].

WHO - World Health Organization (2010) Research Ethics in International Epidemic Response. WHO/ HSE/GIP/ITP/10.1. Available at: https://www.who.int/ethics/gip_research_ethics_.pdf?ua=1 [Last accessed 14 October 2020].

WHO - World Health Organization (2020) Ethical standards for research during public health emergencies: Distilling existing guidance to support COVID-19. WHO/RFH/20.1. Available at: https:// www.who.int/blueprint/priority-diseases/key-action/liverecovery-save-of-ethical-standards-for-resea rch-during-public-health-emergencies.pdf [Last accessed 14 October 2020].

Publisher's Note Springer Nature remains neutral with regard to jurisdictional claims in published maps and institutional affiliations. 